\begin{tabular}{|c|l|}
\hline Title & Convection patterns in a liquid metal under an imposed horizontal magnetic field \\
\hline Author(s) & Y anagisawa, Takatoshi; Hamano, Y ozo; Miy agoshi, Takehiro; Y amagishi, Y asuko; Tasaka, Y uji; Takeda, Y asushi \\
\hline Citation & $\begin{array}{l}\text { Physical Review E, 88(6), 063020 } \\
\text { https://doi.org/10.1103/PhysRevE.88.063020 }\end{array}$ \\
\hline Issue Date & 201312-30 \\
\hline Doc URL & http://hdl.handle.net/2115/66179 \\
\hline Rights & @2013A American Physical Society \\
\hline Type & article \\
\hline File Information & PhysRevE.88.063020.pdf \\
\hline
\end{tabular}

Instructions for use 


\title{
Convection patterns in a liquid metal under an imposed horizontal magnetic field
}

\author{
Takatoshi Yanagisawa, ${ }^{*}$ Yozo Hamano, Takehiro Miyagoshi, and Yasuko Yamagishi \\ Institute for Research on Earth Evolution, Japan Agency for Marine-Earth Science and Technology, Yokosuka, Japan \\ Yuji Tasaka and Yasushi Takeda \\ Graduate School of Engineering, Hokkaido University, Sapporo, Japan \\ (Received 3 September 2013; published 30 December 2013)
}

\begin{abstract}
We performed laboratory experiments of Rayleigh-Bénard convection with liquid gallium under various intensities of a uniform imposed horizontal magnetic field. An ultrasonic velocity profiling method was used to visualize the spatiotemporal structure of the flows with simultaneous monitoring of the temperature fluctuations in the liquid gallium layer. The explored Rayleigh numbers Ra range from the critical value for onset of convection to $10^{5}$; the Chandrasekhar number $Q$ covers values up to 1100 . A regime diagram of the convection patterns was established in relation to the $\operatorname{Ra}$ and $Q$ values for a square vessel with aspect ratio 5 . We identified five flow regimes: (I) a fluctuating large-scale pattern without rolls, (II) weakly constrained rolls with fluctuations, (III) a continuous oscillation of rolls, (IV) repeated roll number transitions with random reversals of the flow direction, and (V) steady two-dimensional (2D) rolls. These flow regimes are classified by the $\mathrm{Ra} / Q$ values, the ratio of the buoyancy to the Lorentz force. Power spectra from the temperature time series indicate that regimes I and II have the features of developed turbulence, while the other regimes do not. The region of steady 2D rolls (Busse balloon) extends to high Ra values in the present setting by a horizontal magnetic field and regime $\mathrm{V}$ is located inside the Busse balloon. Concerning the instabilities of the steady 2D rolls, regime III is the traveling wave convection developed from the oscillatory instability. Regime IV can be regarded as a state of phase turbulence, which is induced by intermittent occurrences of the skewed-varicose instability.
\end{abstract}

DOI: 10.1103/PhysRevE.88.063020

PACS number(s): 47.20.Bp, 47.27.De, 47.65.-d

\section{INTRODUCTION}

The study of the nature of thermal convection in liquid metals under a magnetic field is important to understand the dynamics of planetary metallic cores and in engineering applications such as stable crystal growth and enhancing the efficiency of heat exchangers. An electric current $\mathbf{j}$ is induced when a flow of liquid metal crosses a magnetic field $\mathbf{B}$ and it generates a Lorentz force proportional to $\mathbf{j} \times \mathbf{B}$. The Lorentz force changes the force balance, making the flow behavior different from flows in situations without magnetic fields. Generally, the viscosity of liquid metals is very low and their flow easily becomes turbulent, but when a magnetic field is applied to liquid metals, it enhances two dimensionality and creates anisotropic flow structures suppressing turbulence, depending on the direction and intensity of the field (see [1]).

One of the typical settings to study the effects of a magnetic field on fluid flow is a thermal convection system driven by a vertical temperature gradient, that is, Rayleigh-Bénard (RB) convection. The controlling nondimensional parameters in a $\mathrm{RB}$ convection system under an imposed uniform magnetic field are the Rayleigh number $\left(\mathrm{Ra}=\alpha g \Delta T L^{3} / \kappa \nu\right)$, and the Chandrasekhar number $\left(Q=\sigma B^{2} L^{2} / \rho v\right)$ or the Hartmann number $\left(\mathrm{Ha}=Q^{1 / 2}=B L \sqrt{\sigma / \rho v}\right)$. Here $\alpha$ is the volume coefficient of thermal expansion, $g$ is the gravitational acceleration, $\Delta T$ is the vertical temperature difference between the fluid layer, $L$ is the layer thickness, $\kappa$ is the thermal diffusivity, $v$ is the kinematic viscosity, $\sigma$ is the electrical conductivity, $\rho$ is the fluid density, and $B$ is the intensity of the applied magnetic field. The Rayleigh number expresses the ratio of

\footnotetext{
*yanagi@jamstec.go.jp
}

buoyancy to viscous forces, while the Chandrasekhar number gives the ratio of the magnetic to viscous forces. In addition to Ra and $Q$, liquid metals are characterized by small values of the Prandtl number $(\operatorname{Pr}=\nu / \kappa)$ and the magnetic Prandtl number $(\mathrm{Pm}=v / \eta)$, where $\eta$ is the magnetic diffusivity of the fluid expressed as $\eta=1 / \mu \sigma$, with $\mu$ the magnetic permeability. The values of $\mathrm{Pr}$ and Pm for liquid metals are commonly $10^{-2}$ and $10^{-6}$, respectively. As a result, both the temperature and the magnetic field in liquid metals are more diffusive than the momentum diffusion expressed by $v$.

Theoretical studies on RB convection of liquid metals in horizontally infinite layers [2] indicate that a vertical magnetic field delays the onset of convective motion. The critical Rayleigh number $\mathrm{Ra}_{c}$ increases proportionally to the square of the field strength and the horizontal wave number also grows. In contrast, a horizontal magnetic field does not raise the critical $\mathrm{Ra}$ or change the critical horizontal wave number $k\left(\mathrm{Ra}_{c}=1708\right.$ with $k_{c}=3.117$ for no-slip boundary conditions), but it aligns the axes of convective rolls along the field direction. These theoretical findings are consistent with results of laboratory experiments $[3,4]$.

A series of theoretical studies [5-10] indicate that the parameter regime where two-dimensional rolls remain steady is rather small, in particular for low Pr fluids such as liquid metals. This stable region in the wave number-Rayleigh number space (Busse balloon) is limited by the Eckhaus instability on the smaller wave number side, by the skewedvaricose instability on the larger wave number side, and by the oscillatory instability at $\mathrm{Ra} \sim 1900$ [7]. Without a magnetic field, a convective roll already becomes oscillatory when Ra is increased only slightly beyond its critical value. A horizontal or vertical magnetic field, however, delays the onset of oscillation $[9,10]$. 
The delay of the onset of oscillatory convection by magnetic fields has been confirmed in laboratory experiments and further transitions to chaos or turbulence have been reported [11-17]. However, previous experimental studies lacked the possibility to directly measure flow velocities and therefore have not fully elucidated the convection pattern in the presence of a magnetic field. Most laboratory experiments have been based on pointwise measurements of the temperature in liquid metals. Temperature measurements are suitable for monitoring local fluctuations with high resolutions, but cannot capture larger scale convective structures. The most essential problem needing elucidation is the kind of structural developments in the transition to chaos and turbulence. A broadening of the stable roll region for higher $\mathrm{Ra}$ values under magnetic fields is convenient for detailed experimental studies of these transitions. More information on global patterns of the velocity field have been lacking, holding back progress in understanding liquid metal RB convection.

Visualization of flow patterns is a powerful method to understand flow behavior, but the opacity of liquid metals has been a barrier to observing interior flow phenomena. Originally, laboratory experiments of liquid metal RB convection tracked the movement of grains sprinkled on the surface of mercury $[3,18]$ and this enabled confirmation of the alignment of rolls in the direction of a magnetic field. In Ref. [11], the temperature profile at the top was visualized by a layer of cholesteric liquid crystal, confirming the shape of rolls. Direct measurements of interior flow fields of opaque fluids have been realized in the recent decades by using the ultrasonic velocity profiler (UVP) method. With UVP it is possible to visualize spatiotemporal velocity profiles along ultrasonic beamlines by the Doppler effect. Details of the UVP and recent applications have been reviewed in Ref. [19]. Applications of the UVP to the RB convection of liquid gallium have shown flow patterns and their fluctuations for cases both with and without imposed magnetic fields [20-22]. In general, the UVP can be used for flows with velocities much larger than the detection limit and for RB convection with layer thicknesses of the order of centimeters, which means that the Rayleigh number needs to be several times higher than the $\mathrm{Ra}_{c}$ value, requiring flow velocities of $\sim \mathrm{mm} / \mathrm{s}$. Hence it is difficult at present to detect the onset of convection by the UVP method; however, the UVP is useful to understand developed flow structures with their time variations and the authors here have utilized this advantage.

By measuring flow velocity profiles in a liquid gallium layer, Yanagisawa et al. [21] investigated the gradual suppression of the oscillatory mode when increasing the strength of the imposed horizontal magnetic field. The experiments were performed in a narrow vessel, with a 4:1:1 length ratio; the flow pattern always showed a roll-like structure that was possibly strongly constrained by the sidewalls in the shorter horizontal direction. Flow patterns, free from sidewall constraints, can be expected to emerge in larger horizontal vessels. Yanagisawa et al. [22] used a wider square vessel with a fixed intensity of imposed horizontal magnetic field and observed spontaneous flow reversals characterized by random reversals of the flow direction in the roll structure. Flow reversals were interpreted as a reorganization process of the roll-like patterns moderately constrained by a magnetic field, but the mechanism that induces reversals is not fully understood. The conditions and parameter range for the occurrence of flow reversals is not clear, and the relation to steady roll structures and their instabilities should be elucidated further. This paper extends the studies by [22] for a larger Ra range and various Chandrasekhar numbers $Q$ using the same geometry of square vessel, with simultaneous pointwise measurements of temperature fluctuations in the layer. The aim of the present study is to draw up a regime diagram of flow behavior including the region of flow reversals in relation to $\operatorname{Ra}$ and $Q$ and to give a consistent description of the transition of flow patterns.

\section{APPARATUS AND METHOD}

Figure 1 shows schematic illustrations of the setting of the experiments. The vessel has a square geometry with aspect ratio 5 , which is the same geometry in the previous study [22]. The inside measurements of the vessel are $200 \mathrm{~mm}^{2}$ and $40 \mathrm{~mm}$ deep $(L)$. Liquid gallium is used as the working fluid and the top and bottom plates are made of copper, with the temperature of each plate maintained by circulating water. The sidewalls are 10-mm-thick Teflon, chosen to electrically and thermally insulate these boundaries. A Helmholtz coil system is used to apply a uniform horizontal magnetic field B and its intensity is controlled by a dc power supply. The spatial variance in the magnetic field is within $2 \%$ around the vessel. An UVP is used to measure the velocity field of the flow in the liquid metal and the equipment for the velocity measurements is a UVP-Duo (Met-Flow S.A.); the basic frequency of the ultrasonic transducer is $4 \mathrm{MHz}$. These transducers are set in holes in the Teflon sidewalls and are in direct contact with the liquid gallium. The flow velocities of the gallium are measured along four beamlines from the transducers: uv1 and 2 are set in the direction perpendicular to the magnetic field $\mathbf{B}$, while uv3 and 4 are parallel to $\mathbf{B}$. The diameter of the transducers is $5 \mathrm{~mm}$ and each transducer is located with its center $12.5 \mathrm{~mm}$ away from the bottom (uv1 and 3 ) or top (uv2 and 4) plates. The four transducers are switched on in order for 1.25-s periods, enabling measurements along each line at 5.0-s intervals. The temperature fluctuations in the gallium layer is monitored by a thermistor probe at the middle depth (th1 in Fig. 1), with a sampling rate of $2.0 \mathrm{~Hz}$. The Rayleigh number is controlled by the vertical temperature difference and the maximum value $\Delta T=7.0 \mathrm{~K}$ corresponds to $\mathrm{Ra}=1.5 \times 10^{5}$. The maximum intensity of $\mathbf{B}$ is $18.5 \mathrm{mT}$, which corresponds to the maximum Chandrasekhar number of $1.1 \times 10^{3}$. The Pr and Pm of liquid gallium are 0.025 and $1.5 \times 10^{-6}$, respectively. The calculations of $\operatorname{Ra}, Q, \operatorname{Pr}$, and Pm use the material properties of gallium detailed in Ref. [23]. Further details of the experimental methods are provided in [20,24] and details of the coil system in Ref. [21].

\section{RESULTS}

\section{A. Flow patterns without an imposed magnetic field ( $Q=0$ )}

This section reports the results obtained without imposing a magnetic field, that is, simple Rayleigh-Bénard convection. The Rayleigh numbers in the present experiments are far above the upper boundary of the Busse balloon ( $\mathrm{Ra} \sim 1900$ ) for a nonmagnetic situation [7] and larger than the expected 

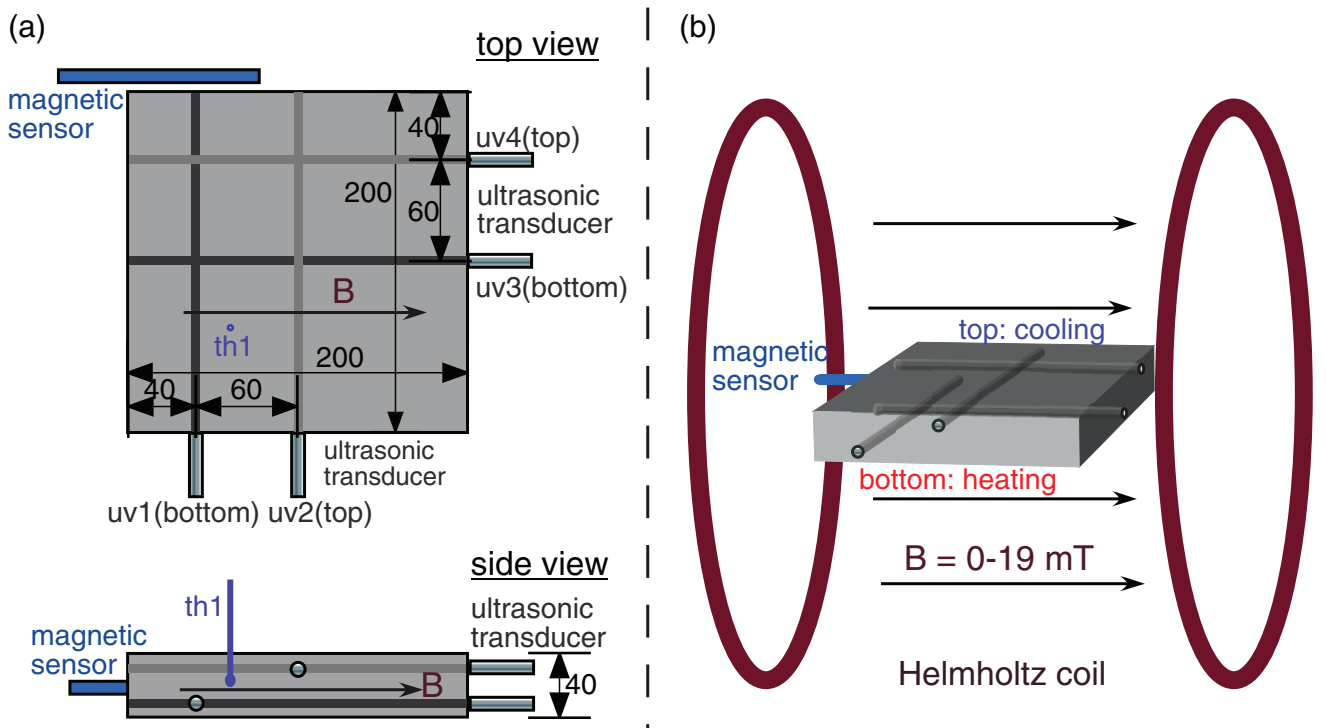

FIG. 1. (Color online) (a) Top and side views of the vessel. The dimensions are in mm. The ultrasonic beamlines uv1 and uv2 measure the velocity profiles perpendicular to the applied magnetic field $\mathbf{B}$, while uv3 and uv4 are parallel to $\mathbf{B}$. Temperature fluctuations in the gallium layer are monitored with a thermistor probe th1. (b) The 600-mm-diam Helmholtz coil and the vessel in the horizontal magnetic field.

transition value to inertial convection $(\mathrm{Ra} \sim 7000)$ with $\mathrm{a}$ time-dependent three-dimensional structure for low Pr fluids [25]. Figure 2 shows the velocity time-space maps of the flow for $2000 \mathrm{~s}$ observed by the UVP at two Rayleigh numbers, $1.5 \times 10^{4}$ [Fig. 2(a)] and $1.5 \times 10^{5}$ [Fig. 2(b)]. The horizontal axis is the time and the vertical axis is the distance from the respective transducers. The UVP captures the component of flow projected on the beamline, hence observing the horizontal velocity along the measurement line. The direction and magnitude of the horizontal flow velocity are displayed in color scale: Blue (minus) indicates flow toward the transducer and red (plus) flow away from the transducer. In both cases, the flow measurements demonstrate that the flow is organized into larger scale cells or structures than the layer thickness scale $L(40 \mathrm{~mm})$. The scale (wavelength) in both directions seems to be similar. These Rayleigh numbers are one and two orders of magnitude higher than the critical value $\left(\mathrm{Ra}_{c}=\right.$ 1708), too high to maintain laminar flow in the low Pr fluid. Hence the observed patterns are fluctuating large-scale cells or a kind of organized flow in turbulence. These fluctuating long-wavelength patterns are consistent with previous studies with low or moderate Pr fluids [26,27] (see the recent review in Ref. [28] for large-scale dynamics in turbulent RB convection). Flow velocities have a similar magnitude in both directions, which suggests that the flow is organized into threedimensional cells rather than quasi-two-dimensional rolls.

A comparison of Figs. 2(a) and 2(b) indicates that flow velocities become larger and fluctuations have shorter time scales at the higher Ra. The Rayleigh number dependence of the representative flow velocity is shown by the closed circles in Fig. 3. Here the representative velocity $U$ is defined as the maximum velocity observed in the second cluster of the velocity profile from the transducer as in Ref. [20]. The value of the measured velocity tends to be smaller at the farther side of the line, which is an artifact arising from the broadening of the ultrasonic beamline and scattering. Hence,
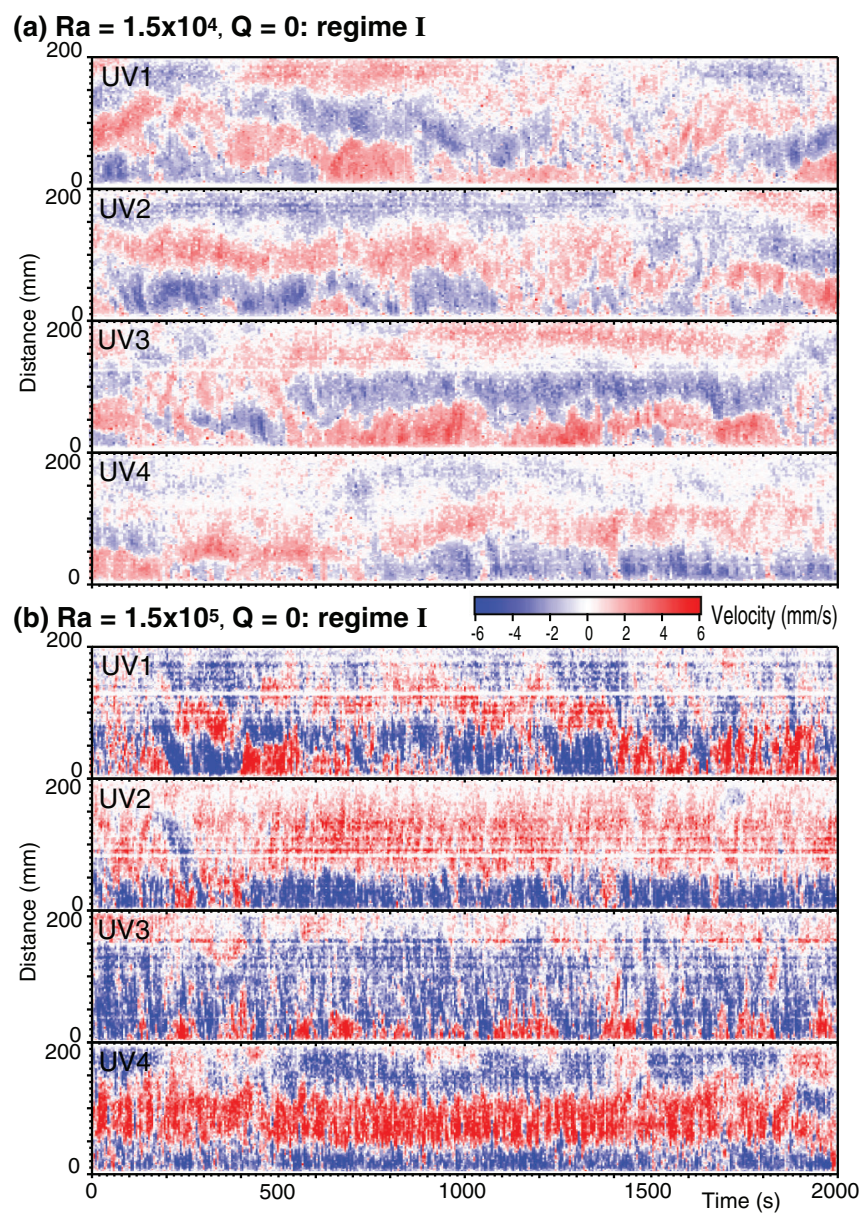

FIG. 2. (Color) Time-space maps of the horizontal flow velocities for $2000 \mathrm{~s}$ with (a) $\mathrm{Ra}=1.5 \times 10^{4}$ and (b) $\mathrm{Ra}=1.5 \times 10^{5}$. The direction and magnitude of the horizontal velocity are shown in the color scale. Here, there is no imposed magnetic field $(Q=0)$ and the flow shows similar velocities in both directions. 


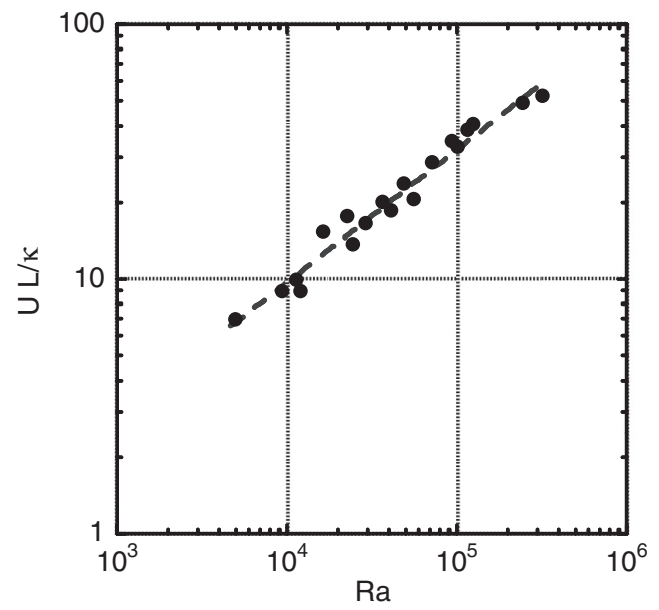

FIG. 3. (a) Plot of the Rayleigh number vs the nondimensional flow velocity without an imposed horizontal magnetic field $(Q=0)$. The gray dashed line is for the relation fitting these points, $U L / \kappa=$ $0.08 \times \mathrm{Ra}^{0.52}$.

to quantitatively compare the flow velocities, the distance from the transducer is fixed. The reasons to adopt the velocity of the second cluster are to prevent the effect of the sidewall on the flow velocity and to prevent the effect of near-field complexity of the ultrasonic beamline from the transducer. The velocity is nondimensionalized by the thermal diffusivity of gallium $\left(\kappa=1.18 \times 10^{-5} \mathrm{~m}^{2} / \mathrm{s}\right)$ and the layer thickness ( $L=40 \mathrm{~mm}$ ). Here $U L / \kappa=10$ corresponds to the velocity of $\sim 3 \mathrm{~mm} / \mathrm{s}$, which is about one order higher than the detection limit of the UVP. These velocity measurements for a series of Ra values in the nonmagnetic cases indicate the dependence on $\mathrm{Ra}$ as $U L / \kappa=0.08 \times \mathrm{Ra}^{0.52}$, which is similar to the result established for a narrower rectangular vessel, $200 \mathrm{~mm}$ long, $50 \mathrm{~mm}$ wide, and $40 \mathrm{~mm}$ high, by [20] $\left(U L / \kappa=0.05 \times \mathrm{Ra}^{0.51}\right)$. These are consistent with a theoretical prediction of the velocity scaling as $\mathrm{Ra}^{1 / 2}$ for low Pr fluids at $\mathrm{Ra}>7 \times 10^{3}$ [25].

Velocity time-space maps by the UVP are essential to establish the time variability of the flow pattern. In contrast, point measurements of the temperature are useful to observe statistic features of the fluctuations. Figure 4(a) shows time series of temperature measurements for $2000 \mathrm{~s}$ at th1 in Fig. 1 for four Rayleigh numbers with $Q=0$. In the experiment, $\mathrm{Ra}$ is increased by increasing the bottom heating temperature with the top cooling temperature kept constant, with the result that the mean temperature and the amplitude of the temperature fluctuations become larger for higher Ra values. Short time scale of fluctuations are observed for higher Ra. Figure 4(b) shows the power spectrum densities (PSDs) calculated from the time series of the temperature in Fig. 4(a). The temperature PSDs are useful to know whether the flow is in developed turbulence. A thermal energy spectrum model for developed turbulence in low Pr fluids [29] predicts a $-5 / 3$ slope for the inertial-convective subrange and a $-17 / 3$ slope for the inertial-conductive subrange in the temperature PSD; these slopes were reported in Ref. [21] for $\mathrm{Ra}>7 \times 10^{4}$ in a narrower vessel without a magnetic field imposed. The dashed lines in the PSD graph show slopes of $-5 / 3$ and $-17 / 3$. All

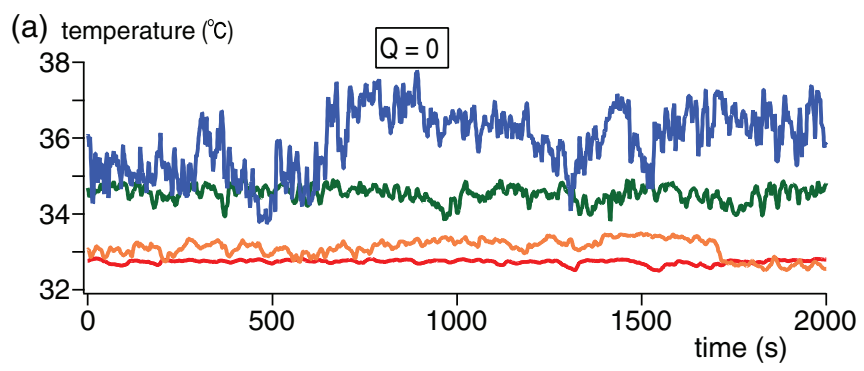

(b) PSD

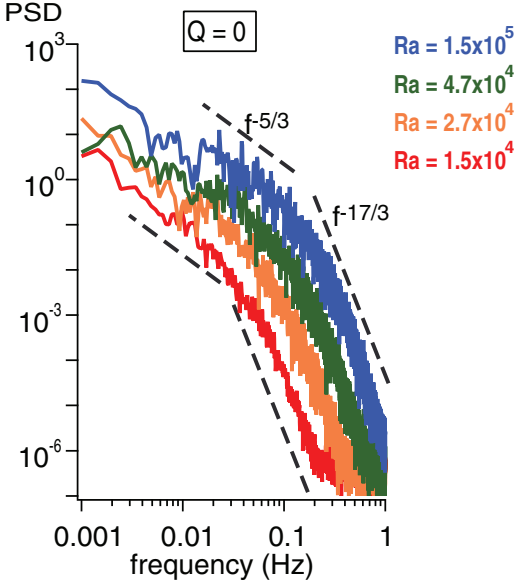

FIG. 4. (Color online) (a) Time series of the temperature and (b) the PSD, for four Ra values without an imposed magnetic field $\mathbf{B}$. The dashed lines in the PSD graph indicate the slopes of $-5 / 3$ (inertial-convective subrange) and $-17 / 3$ (inertial-conductive subrange) expected by the model of developed turbulence for low $\mathrm{Pr}$ fluids.

the PSDs in Fig. 4(b) are in the region fitting these slopes, with the change of slopes more obvious for the higher Ra cases. The frequencies at the slope changes are lower for smaller values of $\mathrm{Ra}$ : around $0.1 \mathrm{~Hz}$ for $\mathrm{Ra}=1.5 \times 10^{5}$ and around $0.03 \mathrm{~Hz}$ for $\mathrm{Ra}=1.5 \times 10^{4}$. These features of the PSDs indicate the presence of the two subranges at these Ra and that the flow in this square vessel with no imposed magnetic field is in a state of developed turbulence.

In summary, without an imposed magnetic field, the flow pattern in the square vessel used here does not have roll-like structures, but has isotropic large-scale cellular circulation for $\mathrm{Ra}>1 \times 10^{4}$. The representative flow velocities are of similar magnitudes in both directions and the dependence on $\mathrm{Ra}$ is $\mathrm{Ra}^{1 / 2}$. The PSD of the temperature fluctuations indicates that the flow is in a state of developed turbulence having an inertial-convective subrange for Ra $>1 \times 10^{4}$. Compared with the results in the narrower vessel in Ref. [21], turbulent flow develops at lower Ra values in this wider square vessel, likely because of the weaker constraint on the flow pattern by the sidewalls.

\section{B. Flow patterns with an imposed horizontal magnetic field $(Q \neq 0)$}

When a horizontal magnetic field is imposed on the vessel (Fig. 1), the flow pattern changes greatly and it is possible to identify five flow regimes, depending on the Chandrasekhar number $Q$ and the Rayleigh number Ra. Figure 5 shows 


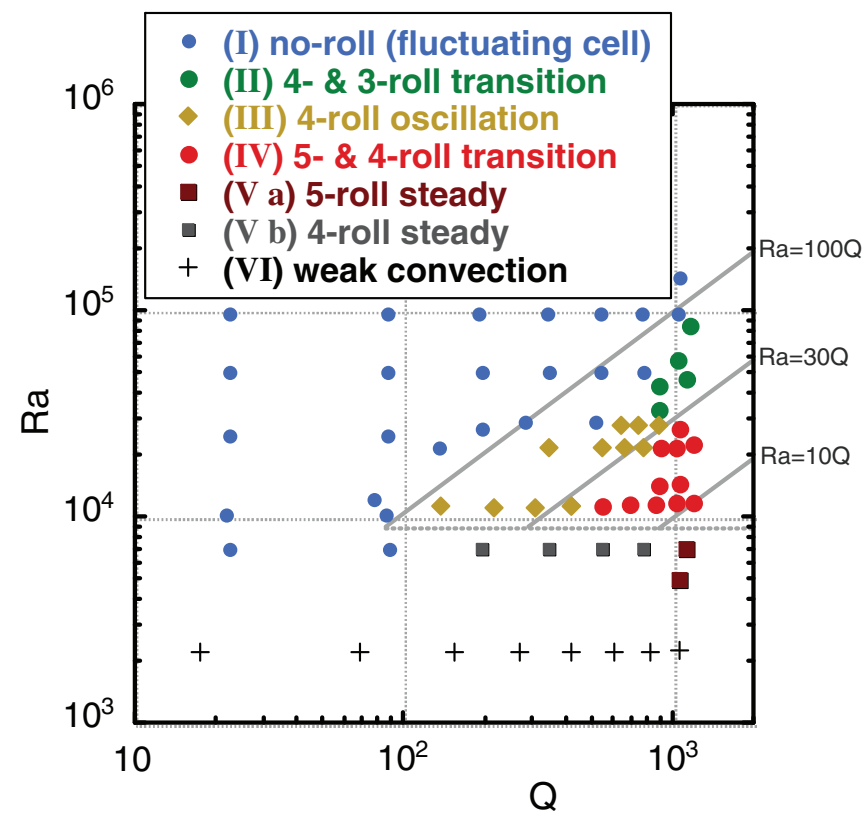

FIG. 5. (Color) Regime diagram of the convection behavior plotting the Chandrasekhar number $Q$ versus the Rayleigh number Ra: regime I, no-roll structures (fluctuating large-scale cells); regime II, transition between four- and three-rolls (weakly constrained rolls); regime III; four-roll structure with continuous oscillation; regime IV, transition between five- and four-rolls including flow reversals; regime $\mathrm{V}$ a, steady flow with five-rolls; regime $\mathrm{V}$ b, steady flow with four-rolls; and regime VI; no ordered flow detectable with UVP. Oblique gray lines suggest isolines of the parameter ratio $\mathrm{Ra} / Q$ and approximately correspond to the regime boundaries.

a regime diagram of the convection behavior in the $Q-\mathrm{Ra}$ plane for this square aspect-ratio-5 vessel. The regimes are classified by the features of the velocity profiles. Regime I is a state with no roll structures (or fluctuating large-scale cells), similar to the state with no imposed magnetic field described in the previous section. Regimes II-V are states showing an effect of the magnetic field with roll-like structures with mean axes parallel to $\mathbf{B}$. Regime II is a state with weakly constrained rolls in which roll number transitions mainly between 4 and 3 are observed. Regime III is a state with continuous oscillations with a four-roll structure. Regime IV is a state where the roll number transitions mainly between 5 and 4 occur intermittently and flow reversals are observed sometimes accompanied by the transitions. This regime IV includes the flow reversals described in Ref. [22], where the parameters were $\mathrm{Ra}=1.5 \times 10^{4}$ and $Q=1.0 \times 10^{3}$. Regime $\mathrm{V}$ is a state with a steady two-dimensional (2D) roll structure: $\mathrm{V}$ a with five-rolls and $\mathrm{V} b$ with four-rolls. Regime VI is used to show the region where it is not possible to observe any flow by the UVP, as the flow velocity is below the detection limit $(\sim 0.3 \mathrm{~mm} / \mathrm{s})$. Fluctuations of temperature by the th 1 probe also could not be detected here for regime VI. Hysteresis in behavior is observed for some points, especially for regimes II and III with an increase and decrease in $Q$. It is noted in detail later in this section. The regime diagram here shows flow behaviors giving priority to regime III.
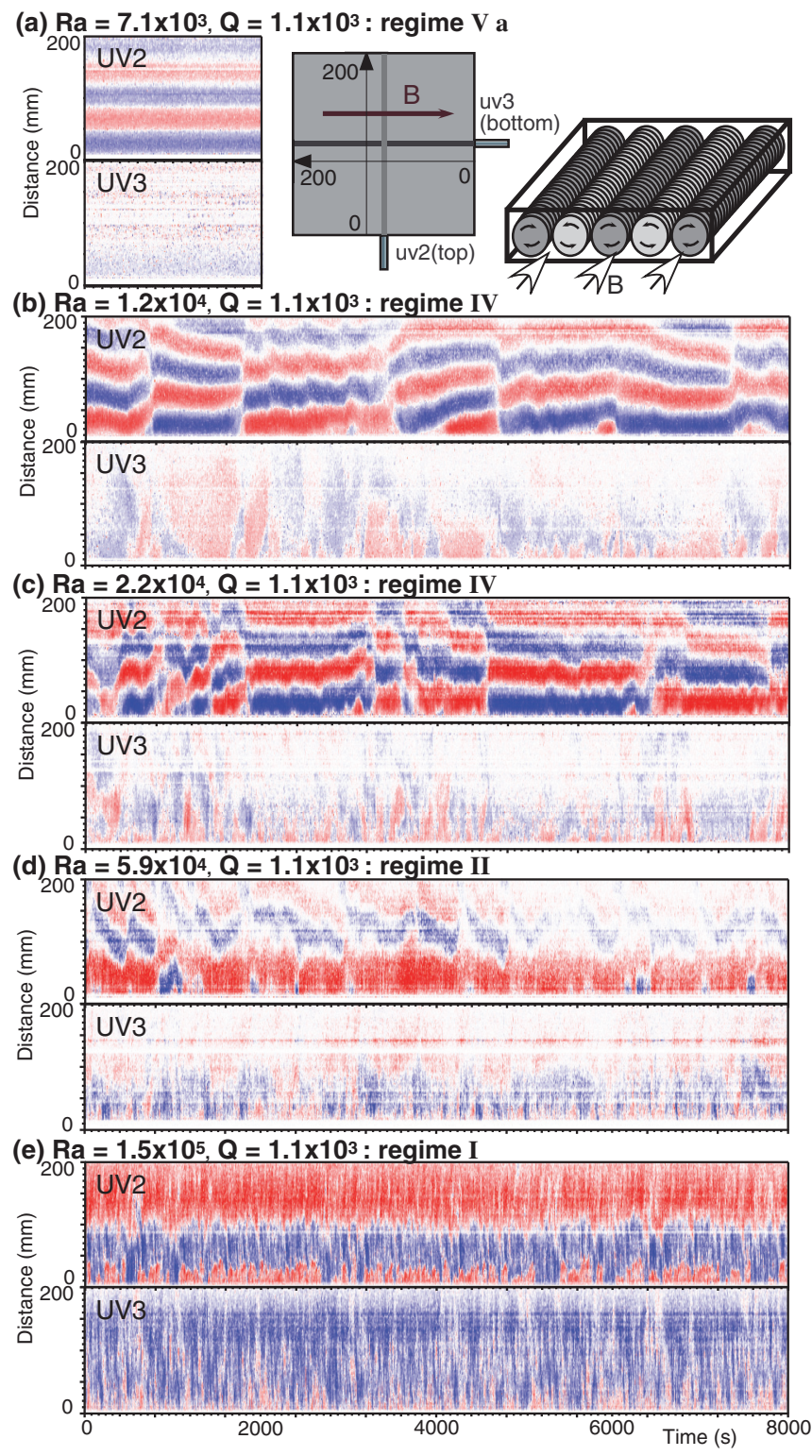

FIG. 6. (Color) Time-space maps of the horizontal flow velocity for (a) $2000 \mathrm{~s}$ and (b)-(e) $8000 \mathrm{~s}$, under a fixed intensity of imposed horizontal magnetic field $\left(B=18.5 \mathrm{mT}, Q=1.1 \times 10^{3}\right.$ ). Velocity profiles for uv2 and uv3 are shown. The velocity color scale is the same as in Fig. 2. The Ra increases from (a) to (e) for (a) regime $\mathrm{V}$ a, steady five-roll; (b) regime IV, transition between five- and four-rolls; (c) also regime IV; (d) regime II, transition between fourand three-rolls; and (e) regime I, no-roll structures. Random reversals of the flow direction are observed in (b) and (c) accompanied by transitions in the number of rolls.

The following explains details of the behavior of the convection in each regime. Figure 6 shows five examples of the velocity measurements. In this figure the intensity of $\mathbf{B}$ is the maximum value with the present apparatus $(18.5 \mathrm{mT}$, $Q=1.1 \times 10^{3}$ ), while Ra is higher for the lower panels. Each experiment is an independent one; the initial condition is an isothermal no-convection state under the indicated intensity of the imposed magnetic field and a specific vertical temperature difference is set once at the start of the experiment to realize 
each Ra. The velocity time-space maps in Fig. 6 show the behavior for $2000 \mathrm{~s}$ [Fig. 6(a)] and $8000 \mathrm{~s}$ [Figs. 6(b)-6(e)], depicting the situation after a thermally balanced state has been attained.

In Fig. 6(a), $\mathrm{Ra}=7.1 \times 10^{3}$ and a steady five-roll structure is clearly observed with the roll axis fixed in the direction of the magnetic field $\mathbf{B}$ (regime $\mathrm{V}$ a). The sign (color) of the velocity in uv2 indicates that the flow is downward near the wall at the distance $0 \mathrm{~mm}$ and upward near the wall at $200 \mathrm{~mm}$. The flow parallel to B (uv3) is very weak. The patterns observed by the other lines uv1 and uv4 conform to this, showing that the flow has a highly regular two-dimensional steady roll structure. The flow structure in the vessel reconstructed from these measurements is schematically shown to the right. This represents a typical flow regime under a strong horizontal magnetic field. There are five rolls showing that the aspect ratio of a roll is $\sim 1.0$, which approximately corresponds to the most likely wave number (3.117) near the critical Ra with a no-slip boundary condition for a horizontally infinite layer [2]. That is, using the number of rolls $n$ observed in this vessel and the layer thickness $L$, the (dimensionless) wave number is defined as $\pi n /(5 L / L)$. The wave numbers for this steady five-roll structure in the directions perpendicular and parallel to $\mathbf{B}$ are $\pi$ and 0, respectively. The other examples of velocity measurements in Fig. 6 show time-dependent behaviors.

Figure $6(\mathrm{~b})$ is for $\mathrm{Ra}=1.2 \times 10^{4}$ and is classified into regime IV. The flow velocity in the direction perpendicular to $\mathbf{B}$ is much larger than in the direction parallel to $\mathbf{B}$, which shows the existence of a roll-like structure, while transitions in the roll number between 5 and 4 occur at irregular time intervals. Reversals of the flow direction in the rolls sometimes occur accompanied by the roll number transitions (e.g., at around $800,1800,3600$, and $7500 \mathrm{~s}$ ). In this time series [Fig. 6(b)], a five-roll structure is dominant and the average duration of one pattern (interval between transitions) is $\sim 400 \mathrm{~s}$. Figure $6(\mathrm{c})$ for $\mathrm{Ra}=2.2 \times 10^{4}$ is also classified into regime IV with roll number transitions between 5 and 4 as well as flow reversals, but with more fluctuations than in Fig. 6(b). A four-roll structure is dominant in Fig. 6(c) and the durations of a pattern tends to be shorter than in Fig. 6(b). Examples of regime III are presented separately later, as regime III is not observed for this value of $Q$.

Figure $6(\mathrm{~d})$ is for $\mathrm{Ra}=5.9 \times 10^{4}$ and classified into regime II, showing roll number transitions between 4 and 3 for the uv 2 profile. The pattern maintains a roll-like structure, but the three dimensionality of the flow has increased. The flow velocity for uv2 is higher than for uv3, but the difference is not as clear as in Figs. 6(b) and 6(c). A three-roll structure is dominant in Fig. 6(d), with a fourth roll-like structure intermittently emerging near the sidewalls, with only short lifetimes. Flow reversals are not clearly observed in this regime.

Figure $6(\mathrm{e})$ is for $\mathrm{Ra}=1.5 \times 10^{5}$ and classified into regime I, where whole- or half-vessel scale flows are observed with short time fluctuations and the magnitude of the velocity similar in both directions. There are no roll-like structures in Fig. 6(e), though the experiment takes place with a horizontal magnetic field imposed. The behavior in Fig. 6(e) is similar to that without a magnetic field imposed for the same Ra shown in Fig. 2(b).
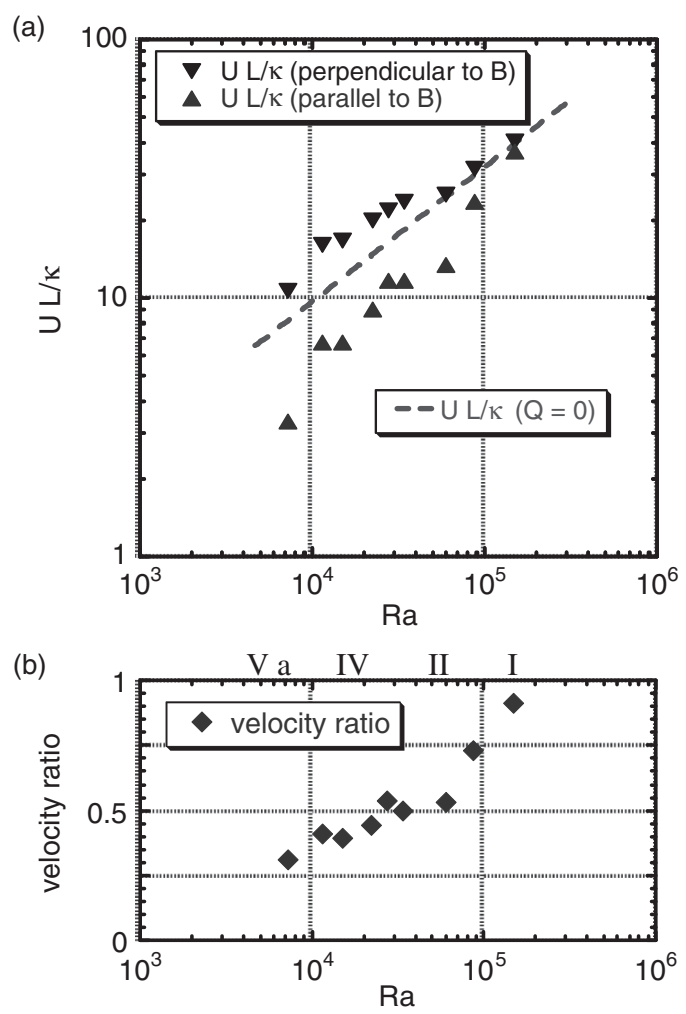

FIG. 7. (a) Plot of the Rayleigh number vs the nondimensional flow velocity with an imposed horizontal magnetic field of $18.5 \mathrm{mT}$ $\left(Q=1.1 \times 10^{3}\right)$. The vertically aligned pairs of triangles indicate the velocity in the directions perpendicular to and parallel to $\mathbf{B}$. The gray dashed line is the fitting line from Fig. 3 with the nonmagnetic situation. (b) Ratio of the velocity parallel to $\mathbf{B}$ to the velocity perpendicular to $\mathbf{B}$, calculated from the values in (a) for the various Ra values.

The features of velocity time-space maps observed here are analyzed quantitatively. The relation between $\mathrm{Ra}$ and representative flow velocity is presented in Fig. 7(a) for imposed magnetic field $B 18.5 \mathrm{mT}\left(Q=1.1 \times 10^{3}\right)$. Pairs of downward and upward triangles for various $\mathrm{Ra}$ values represent the flow velocities perpendicular and parallel to $\mathbf{B}$. In all the cases presented in Fig. 7, the flow velocities in the direction perpendicular to $\mathbf{B}$ are larger. The representative velocity is determined in the same manner as in the nonmagnetic field case (Fig. 3): the maximum velocity observed in the second cluster of the same flow direction from the transducer. In cases where the flow structure is not clearly discernible for the velocity parallel to $\mathbf{B}$ (especially at lower $\mathrm{Ra}$ values), the average velocity at around $75 \mathrm{~mm}$ from the transducer is adopted. Figure 7(b) shows the ratio of the velocity values (parallel to $\mathbf{B}) /($ perpendicular to $\mathbf{B}$ ) versus $\mathrm{Ra}$. This ratio reflects the degree of anisotropy and is expected to be 1.0 for isotropic flow structures. Anisotropic velocities dominate at lower Ra values, which means that there is a strong two dimensionality in the flow structure. Figure 7(b) indicates that the degree of anisotropy in the velocity decreases with growing $\mathrm{Ra}$. The velocities in both directions are very similar for $\mathrm{Ra}>10^{5}$ under the imposed magnetic field corresponding to $Q=1.1 \times 10^{3}$, where the pattern is classified into regime $\mathrm{I}$. 


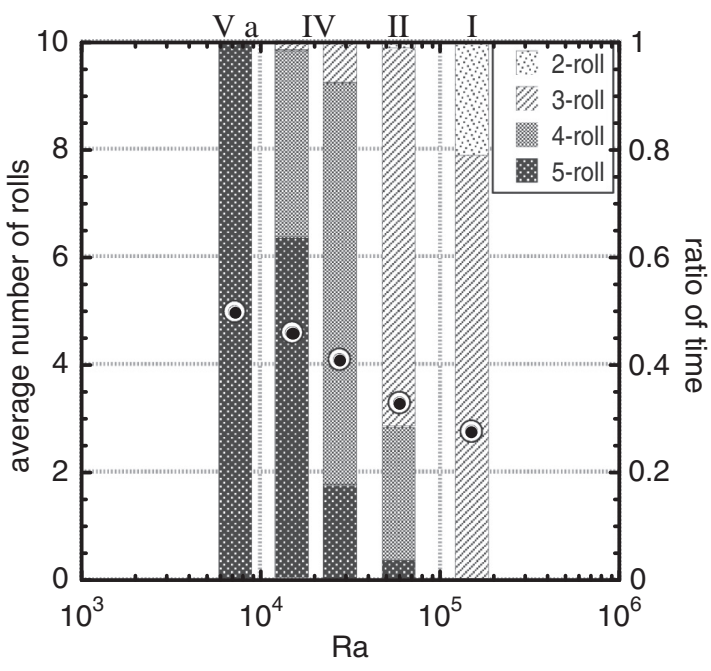

FIG. 8. Plot of the Rayleigh number vs the ratios of the observed times for each number of rolls (columns, right axis) and the average of the number of rolls (concentric circles, left axis). There is an imposed horizontal magnetic field of $18.5 \mathrm{mT}\left(Q=1.1 \times 10^{3}\right)$.

Figure 8 shows the Ra dependence of the number of rolls observed in the direction perpendicular to B. From the time-space maps for uv2, we converted the data of the durations with the number of rolls (or number of clusters for the same flow direction). For example, in Fig. 6(b), it is a five-roll structure for time $0-740 \mathrm{~s}$, a four-roll structure for $740-890 \mathrm{~s}$, a five-roll structure for $890-1780 \mathrm{~s}$, and so on. The total durations of the time series used for the analysis are 12000-20000 s in the fluctuating cases and each case includes more than 20 roll number transitions. The ratio of time for a number of rolls is plotted against Ra by the columns graph in Fig. 8 (right vertical axis), with the magnetic field $18.5 \mathrm{mT}\left(Q=1.1 \times 10^{3}\right)$ here. At Ra $=7.1 \times 10^{3}$, the pattern is a steady five-roll structure (regime $\mathrm{V}$ a), so the ratio of time with five-rolls is $100 \%$. At Ra $=1.2 \times 10^{4}$, the five-roll structure occurs for more than $60 \%$ of the whole duration and the rest is a four-roll structure. At Ra $=2.2 \times 10^{4}$, there are some five-roll structures, while the four-roll structure is dominant. The results with these two Ra values both belong to regime IV. At Ra $=5.9 \times 10^{4}$ (regime II), the three-roll structure is the dominant pattern (70\%) with $25 \%$ of four-roll structures. Above $\mathrm{Ra}=10^{5}$, there is no roll structure, hence the ratio of time is not for the number of rolls but for the number of clusters with the same flow direction observed in uv2. The double circles in each column indicate the average number of rolls; the value is indicated on the left side vertical axis. This plot shows a clear decreasing trend in the average number of rolls with the increase in Ra. Five rolls correspond to the value expected by linear stability theory as the critical size at the onset of convection. These data indicate that, though the behaviors appear complex with many fluctuations, the time-averaged structure is relatively simple and can be described by a monotonic expansion of the horizontal scale of the pattern with increases in the Ra.

The closed circles in Fig. 9 plot the mean durations between pattern transitions at each Ra value belonging to regimes IV and II, with $Q=1.1 \times 10^{3}$. The vertical bars indicate the

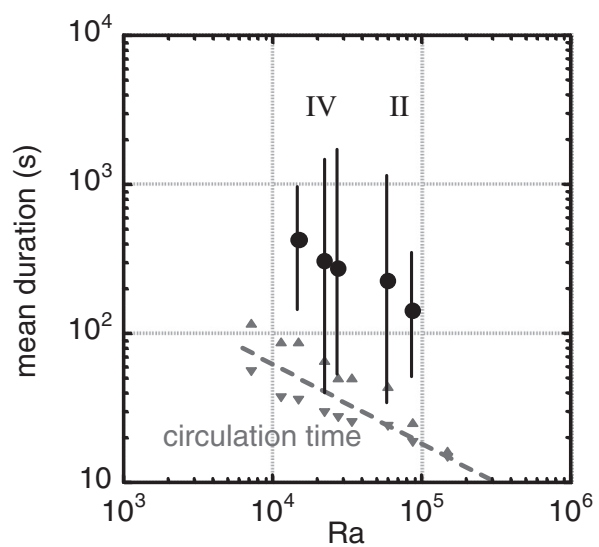

FIG. 9. Plot of the Rayleigh number vs the mean duration of flow patterns (black circles). Bars extending from the circles indicate the range of maximum and minimum durations observed at a Ra. The circulation times estimated from the flow velocities in Figs. 3 and 7 are indicated by a gray dashed line and gray triangles. The imposed horizontal magnetic field here is $18.5 \mathrm{mT}\left(Q=1.1 \times 10^{3}\right)$.

ranges of the maximum and minimum durations observed for a Ra value. Though the time variability is very large, the mean duration decreases monotonically with increases in Ra. Pattern transitions and the resulting flow reversals appear to occur randomly, while the mean durations show a clear relation to Ra. The dashed line and triangles in Fig. 9 indicate circulation times at the various $\mathrm{Ra}$ values. These circulation times (typical flow time scales) are approximate estimates using the flow velocity shown in Figs. 3 and 7(a) by assuming that the circulation path is equal to the length of a roll with aspect ratio one $(\sim 160 \mathrm{~mm})$ and that the vertical flow velocity is equivalent to the measured horizontal flow velocity. The dashed line represents the fitting for the $U L / \kappa$ noted in the previous section for $Q=0$. The data for this in Fig. 9 indicate that the mean durations of the pattern transitions have a time scale of one order longer than the circulation times estimated by the flow in the direction perpendicular to $\mathbf{B}$ (downward pointing triangles). The average durations have much longer time scales than the circulation times, but their relation to $\mathrm{Ra}$ is similar $\sim \mathrm{Ra}^{-1 / 2}$. This indicates that the pattern transitions are closely related to the circulation of the flow.

Time series of the temperature measurements and their PSDs for four Rayleigh numbers are show in Fig. 10, with a $B$ of $18.5 \mathrm{mT}\left(Q=1.1 \times 10^{3}\right)$. The UVP measurements indicate that $\mathrm{Ra}=1.5 \times 10^{4}$ and $2.7 \times 10^{4}$ belong to regime $\mathrm{IV}, \mathrm{Ra}=4.7 \times 10^{4}$ to regime II, and $\mathrm{Ra}=1.5 \times 10^{5}$ to regime I. Comparing with the $Q=0$ time series in Fig. 4(a), there appears to be a smaller temperature fluctuation for $Q=$ $1.1 \times 10^{3}$ in the time series for $\mathrm{Ra}=1.5 \times 10^{4}, 2.7 \times 10^{4}$, and $4.7 \times 10^{4}$. While with $\mathrm{Ra}=1.5 \times 10^{5}$, there is no depression of the temperature fluctuations. These changes are clearly shown in the PSDs. The form of the PSD for Ra $=1.5 \times 10^{5}$ is similar to that with $Q=0$ [Fig. 4(b)], having regions fitting the $-5 / 3$ and $-17 / 3$ slopes. The reduction of power under the imposed magnetic field is more dominant for smaller $\mathrm{Ra}$ values. The transition of the PSD slopes also becomes less pronounced at smaller $\mathrm{Ra}$ values. With $\mathrm{Ra}=4.7 \times 10^{4}$ the two subranges are still distinct, while the PSD for $1.5 \times 10^{4}$ 


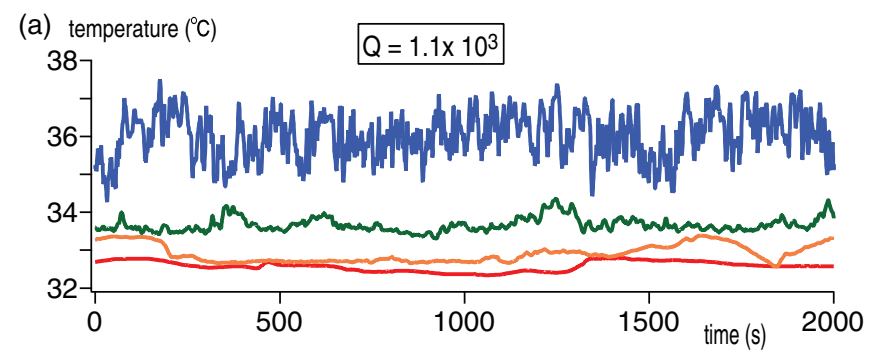

(b) PSD

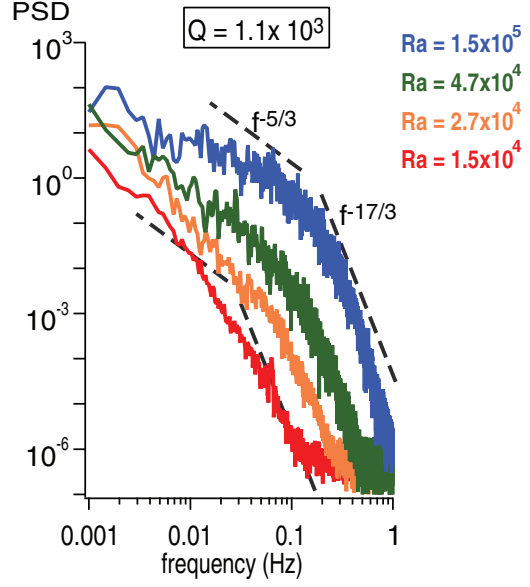

FIG. 10. (Color online) (a) Time series of the temperature and (b) the PSD, for four Ra values with a $B$ of $18.5 \mathrm{mT}\left(Q=1.1 \times 10^{3}\right)$. The dashed lines in the PSD are the same as in Fig. 4(b). The two subranges are clearly observable for the higher Ra values, while there is no clear break for the lower Ra values.

has a single slope with no clear break. These results correspond to the different states of turbulence with the enhancement of two dimensionality of the flow for the lower Ra values. Overall the results here support the conclusion that the PSDs for the flow in regimes I and II show the features of developed turbulence with an inertial-convective subrange, while the PSDs for regime IV do not display distinct subranges. This feature of the temperature PSD and the behaviors of the flow patterns by the velocity profiles indicate that regime IV is a state that may be termed spatiotemporal chaos (see $[30,31]$ ), an intermediate step toward fully developed turbulence.

Regime boundaries are not strictly definitive and hysteresis is observed for the transitions between regimes. This is noticeable in the emergence of regime III, which is an oscillatory state maintaining a four-roll structure. Regime III is mainly observed when $B$ is decreased from a large value where the pattern located in regime IV, with roll number transitions between 5 and 4. On the regime diagram (Fig. 5), some of the points for regime III changed to regime II if the value of $Q$ was increased from regime I.

Figure 11 shows an example of the process of regime III emergence in a continuous experiment with stepwise decreases in $Q$, ordered from top to bottom at one $\mathrm{Ra}$ value $(\mathrm{Ra}=1.2 \times$ $\left.10^{4}\right)$. Figure 11 (a) $\left(Q=5.4 \times 10^{2}\right)$ belongs to regime IV with a flow reversal occurring at about 1200 s, while Fig. 11(b) $\left(Q=3.1 \times 10^{2}\right)$ shows a continuous four-roll structure with small amplitudes of oscillation (regime III). In Fig. 11(c) $(Q=$ $\left.1.3 \times 10^{2}\right)$, the pattern maintains a four-roll oscillatory state (regime III) with larger amplitudes. When the value of $Q$ was
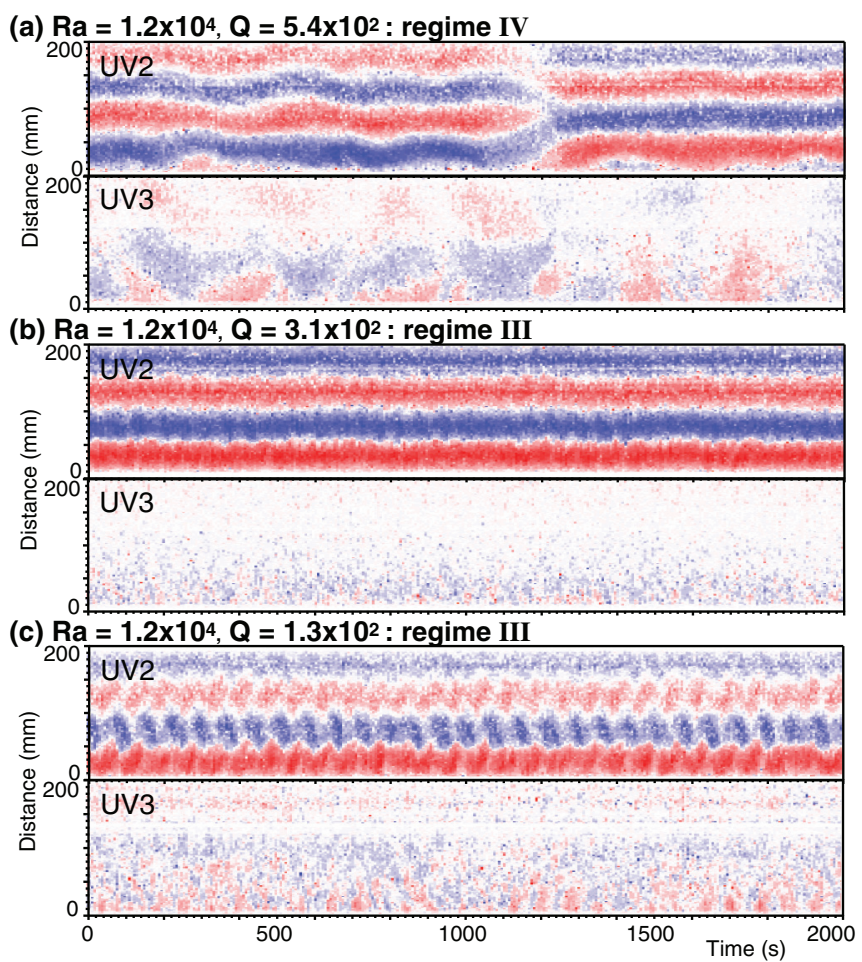

FIG. 11. (Color) Time-space maps of the horizontal flow velocities for $2000 \mathrm{~s}$ with stepwise decreases in the imposed horizontal magnetic field. Velocity profiles for uv2 and uv3 are shown. The velocity color scale is the same as in Fig. 2. The Rayleigh number is $1.2 \times 10^{4}$; (a) regime IV, transition between five- and four-rolls with a flow reversal at $1200 \mathrm{~s}$; (b) regime III, four-roll structure with small oscillations; and (c) also regime III with larger amplitude of oscillations.

reduced to $\sim 80$, the pattern changed to a no-roll structure that is classified into regime $\mathrm{I}$.

The same oscillation time scales detected in the velocity measurements for regime III are also clearly observed in the temperature measurements. Figure 12(a) shows a time series of temperatures for the three cases in Fig. 11. For $Q=3.1 \times$ $10^{2}$ and $1.3 \times 10^{2}$, the periodic behaviors in the temperature reflect the oscillations of the four-roll structure [Figs. 11(b) and 11(c)], while the global pattern transitions induce large amplitudes of temperature fluctuations with $Q=5.4 \times 10^{2}$. Figure 12(b) shows the PSDs of the temperatures calculated from these time series. The PSDs for $Q=3.1 \times 10^{2}$ and $1.3 \times$ $10^{2}$ have sharp peaks, a typical feature of the flow in regime III. For smaller values of $Q$, the frequency of peaks is lower and the power is higher. The slopes of $-5 / 3$ and $-17 / 3$ are also indicated in the graph as in Figs. 4 and 10, but corresponding slopes are not visible in these PSDs.

Figure 12(c) shows the dependences of $Q$ on the oscillation period and oscillation range for regime III at $\mathrm{Ra}=1.2 \times 10^{4}$. The oscillation period (circles, right vertical axis) is the inverse of the peak frequency with the maximum power in the temperature PSD. The oscillation range (diamonds, left vertical axis) is the range of movement of the zero-velocity position in the second roll from the transducer in the UVP measurements. As far as the oscillatory behavior of the four-roll situation is observed, the period increases slightly with decreases in $Q$ and 


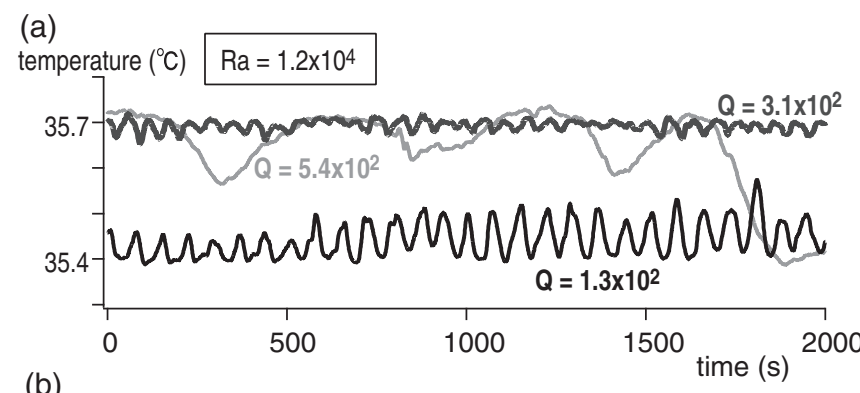

(b)

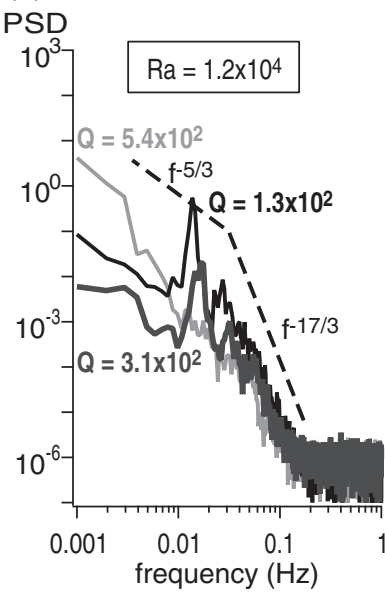

(c)

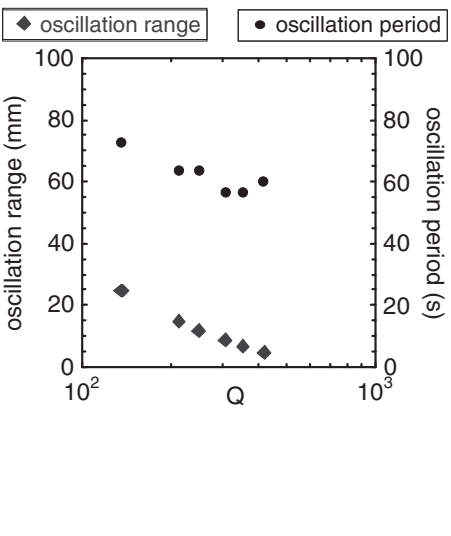

FIG. 12. (a) Time series of the temperatures and (b) the PSD for three intensities of imposed magnetic fields with $\mathrm{Ra}=1.2 \times 10^{4}$. There are distinct peaks of power for $Q=3.1 \times 10^{2}$ and $1.3 \times 10^{2}$. (c) The left axis shows $Q$ vs the range of oscillation observed by the UVP and the right axis shows $Q$ vs the peak period in the temperature PSD. All the data in (c) fall in regime III.

simultaneously the spatial range of its horizontal fluctuation gets larger. This corresponds to the relative weakening of the restriction imposed by the horizontal magnetic field, causing the increase of velocity fluctuations in both directions. The typical period of oscillation is 60-70 s for the cases here. As the circulation time of the flow for a roll is 30-40 s (the length of one circulation path for a four-roll structure is $\sim 180 \mathrm{~mm}$ and the typical flow velocity perpendicular to $\mathbf{B}$ is $5-6 \mathrm{~mm} / \mathrm{s}$ ), this oscillation period is about two times the circulation time. As shown in the regime diagram, similar oscillatory four-roll structures were observed for the series of experiments at $\mathrm{Ra}=2.2 \times 10^{4}$ and $2.8 \times 10^{4}$, with decreases in $Q$ from regime IV.

On the regime diagram (Fig. 5), isolines of the parameter ratio $\mathrm{Ra} / Q$ are also shown. These lines suggest that the classification of flow regimes above $\mathrm{Ra} \sim 10^{4}$ can be understood by the values of $\mathrm{Ra} / Q$; the boundary line for regime I (no-roll regime) corresponds to $\mathrm{Ra} / Q \sim 100$ and regime IV (roll number transition mainly between 5 and 4 , including flow reversals) is divided by the two lines $\mathrm{Ra} / Q \sim 30$ and $\mathrm{Ra} / Q \sim 10$. From the definitions of $\mathrm{Ra}$ and $Q, \mathrm{Ra} / Q$ means the ratio of the buoyancy to the Lorentz force. Hence the upper left side of the diagram is the region where the buoyancy is much larger than the Lorentz force, while the lower right side is the region where the Lorentz force is dominant. In the region where $\mathrm{Ra} / Q>100$, the effect of the applied magnetic field

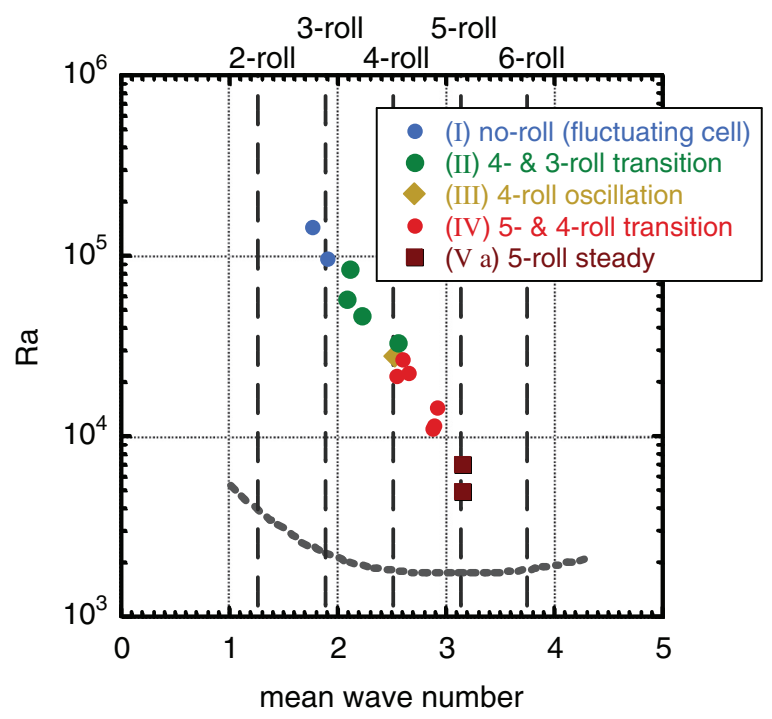

FIG. 13. (Color) Plot of mean wave number vs Rayleigh number for $Q=870-1100$. The mean wave number is the time-averaged number of rolls observed in the uv2 profiles. The marks for the regimes are the same as in Fig. 5. The dotted line at the bottom of the graph is the neutral curve for the onset of convection $\left(\mathrm{Ra}_{c}\right)$ with a no-slip boundary, in a horizontally infinite layer.

is not visible on the flow behavior; isotropic structures are dominant and the flow is in the developed turbulence.

\section{DISCUSSION}

The experiments here indicate that the variations in the flow regimes are accompanied by expansions in the horizontal scale of rolls pointing toward developed turbulence, that is, a reduction of the wave number of a pattern in the direction perpendicular to $\mathbf{B}$. Figure 13 summarizes the relation between the time-averaged wave number and $\mathrm{Ra}$ for $Q$ values between 870 and 1100. The wave numbers are calculated from the velocity profiles of uv2. In this graph, five points coincide with the data on the average number of rolls in Fig. 8. The graph shows a monotonic decreases of the time-averaged wave number toward higher $\mathrm{Ra}$ values through all five flow regimes. When $\mathrm{Ra}$ is small and the effect of the magnetic field is sufficiently strong, the pattern is maintained in the steady $2 \mathrm{D}$ roll state (regime $\mathrm{V}$ a). As the relative intensity of the buoyancy increases, more complex spatial patterns and temporal behaviors are encountered. The increase in the horizontal scale with $\mathrm{Ra}$ causes incongruities in the roll size with the fixed geometry of the vessel. The discrepancy between the intrinsic roll size and the vessel size may be a factor in inducing the repeating transitions in the number of rolls and flow reversals (regime IV). At the Ra where the intrinsic roll size with fluctuations matches the vessel, the oscillatory state of the four-roll structure is maintained for a long period (regime III). For higher Ra, a structure with a smaller wave number (three-roll) arises, with increased velocities in the direction parallel to $\mathbf{B}$, and the roll-like structure becomes less clear (regime II). For $\mathrm{Ra}>10^{5}$, an almost isotropic flow is attained with three or two velocity clusters in both directions (regime I). 
The situation that the wave number of patterns decreases as $\mathrm{Ra}$ increases is commonly observed in simple (nonmagnetic) RB convection with various fluids of $\operatorname{Pr}>0.7$ [32,33]. Figure 13 indicates that liquid metal convection $(\operatorname{Pr}=0.025)$ is subject to a similar feature under a fixed intensity of an imposed horizontal magnetic field.

The delay of the onset of oscillatory convection by a horizontal magnetic field was studied by a numerical analysis [10] and a laboratory experiment [13]. In Ref. [10], it was reported that the regime for stable two-dimensional rolls (Busse balloon) is extended from $\mathrm{Ra} \sim 1900$ to $\sim 2100$ with $\operatorname{Pr}=0.025$ when $Q=30$. In Ref. [13], the oscillatory instability onset was measured in rectangular vessels up to $Q \sim 70$ (then oscillation onset at $\mathrm{Ra} \sim 3500$ ). These $Q$ values are too small to compare directly with the present result, however, the transitions between the regimes observed here can be discussed based on the Busse balloon concept. The first-order effect of the horizontal magnetic field is obviously to extend the Busse balloon to higher Rayleigh numbers. The steady roll in regime $\mathrm{V}$ of the present experiment is located inside the Busse balloon, which may be extended to $\mathrm{Ra} \sim 10^{4}$ by the imposed horizontal magnetic field $Q \sim 10^{3}$. This value is consistent with the simple extrapolation of the relation in Ref. [13] for the onset of oscillation ( $\mathrm{Ra} \sim 15000)$, though the relation was made by the fitting for $Q<70$. The flow velocities are below the detection limit of the UVP in the present vessel for $\mathrm{Ra}<5000$. The larger $Q$ values used here extend the roll-like regime to even larger $\mathrm{Ra}$ and thus detectable flow velocities. It may be possible to detect the oscillation onset for $10^{2}<Q<10^{3}$, by the measurement of temperature fluctuations in the present vessel, and to connect the range of these experiments.

The oscillation of the four-roll structure (regime III), which is located at larger $\mathrm{Ra}$ values for the steady roll mode, thus seems equivalent to the oscillatory instability reported in Ref. [6]. This regime corresponds to the traveling wave convection [34] over the $\mathrm{Ra}$ at the oscillatory instability boundary. The following are the features of the four-roll oscillation observed in the present experiments: (1) The wave number in the direction perpendicular to $\mathbf{B}$ is 2.51 (four-roll) and uv3 in Fig. 11(c) suggests that the wave number in the direction parallel to $\mathbf{B}$ is smaller than this (having a longer wavelength with approximately three clusters). (2) The period of the oscillatory motion is related to the circulation time of a roll. This is consistent with the structure of the traveling wave convection reported in Ref. [34], though the flow in the present experiment is restricted by sidewalls. In a laboratory experiment [14], traveling waves were observed by a lattice of thermistors above the oscillatory instability ( $\mathrm{Ra} \sim 3000-4000$, $Q \sim 10^{2}$ ). Also, large hysteresis was reported in the transition between steady and oscillatory convection, with an increase and decrease of $\mathrm{Ra}$. The present result of regime III shows features common with those of [14] and may correspond to the same behavior at larger $\operatorname{Ra}$ and $Q$ values.

Regime IV, the repeated transitions between five- and four-rolls, is located adjacent to the region of steady and oscillatory rolls, which suggests that it has developed as a result of the skewed-varicose instability of the five-roll steady structure. As the boundary of the skewed-varicose instability prevents the steady roll region from extending in the larger wave number direction [10], the time-averaged wave number may become smaller with increases in Ra as shown in Fig. 13. It is possible to distinguish the skewed-varicose instability (instability in both directions perpendicular and parallel to the axis of roll) and the Eckhaus instability (pure 2D instability with variations in the wave number perpendicular to the axis of roll) by a comparison of two velocity profiles set in the same direction. The velocity measurements of uv1 and uv2 indicate that there is a time lag in the transitions and slight differences in the profiles along the axis of the rolls (see Fig. 2 in Ref. [22]). This reflects the skew of the roll-like structure in the direction of the magnetic field. Regime IV can be considered a case of repeated skewed-varicose instability because the roll number transitions occur spatially and intermittently as observed in these velocity profiles. This also suggests that regime IV is the kind of state termed phase turbulence (e.g., [33,35]). In general, phase turbulence in $\mathrm{RB}$ convection is assumed to occur in a horizontally wide layer with free-free boundary conditions for low Pr fluids and to emerge as a result of the skewedvaricose instability [36-38]. It may be possible for this to occur in the present setting (moderate aspect ratio of the layer, with no-slip boundary conditions) because of the relatively high $\mathrm{Ra}$ values with substantial delays in the transition to developed turbulence by the imposed horizontal magnetic field together with the forced orientation of the roll-like structure.

A stability analysis for a horizontally finite layer with sidewalls in Ref. [17] indicated that the horizontal magnetic field raises the critical $\mathrm{Ra}$ for onset of convection depending on the horizontal scale of the layer because of the emergence of sidewall Hartmann layers. This is in contrast to the situation for a horizontally infinite layer in Ref. [2]. The rise of $\mathrm{Ra}_{c}$ at large $Q$ values was confirmed by laboratory experiments in the same paper [17]. Their stability analysis also predicts a slight variation in the critical wave number. By applying their analysis to the present geometry with $Q \sim 10^{3}$, the critical $\mathrm{Ra}$ and the critical wave number rise to $\sim 2500$ and to $\sim 3.3$, respectively. The rise of $\mathrm{Ra}_{c}$ is not so large as to be detectable in the present experiments. In the case of larger $Q$ values, the effect of the increase in $\mathrm{Ra}_{c}$ should be included and some modifications to the specific values of $\mathrm{Ra} / Q$ for regime classifications may be necessary. The rise of the critical wave number to 3.3 may be the cause of the emergence of a five-roll structure at $Q \sim 10^{3}$ in the regime diagram (Fig. 5). The difference in $\mathrm{Ra}_{c}$ for the wave number of 2.5-3.5 is very small for a horizontally infinite layer, as shown by the neutral curve in Fig. 13. This indicates that four-roll and five-roll structures have similar chances of emergence at the onset of convection with small $Q$. When the layer is confined by the sidewalls, the critical wave number to realize the minimum $\operatorname{Ra}_{c}$ shifts slightly to a larger value with the increase in $Q$. That may cause the increase in the number of rolls from 4 to 5 for steady roll convection. The results of the laboratory experiment is mainly for $\mathrm{Ra} / Q \ll 1$ with very large $Q \sim 10^{6}$ [17], having no overlapping region with the present experiment except for nonmagnetic cases. However, behaviors observed in the present experiment have features common with that by temperature measurements. The flow direction deduced by the four-element temperature probe in the experiment in Ref. [17] is isotropic when $Q$ is zero. The 
present result by direct measurements of flow velocity for $Q=0$ is consistent with that. With an increase in $Q$, [17] reported the decrease of temperature fluctuation, emergence of periodic oscillation, increase of anisotropy of flow direction, and increase of Nusselt number (heat flow). These results suggest the transition of flow pattern to aligned roll structure in the direction of $\mathbf{B}$ with suppression of turbulence, as observed in the present experiments. To connect the regions of these two studies, further experiments are expected with $Q \sim 10^{4}-10^{5}$ in the present setting.

The upward direction in this regime diagram, which is the increase in $\mathrm{Ra}$, is the decreasing direction of the wave number as shown by Fig. 13 and it is the direction destabilizing the roll-like structure. The leftward direction, which is the decrease in $Q$, is also the direction of the number of rolls decreasing with the reduction of stability of the roll-like structure. Though the steady roll structure is observed for $\mathrm{Ra}<10^{4}$ in the present experiment, if the maximum value of $Q$ can be raised in this vessel, the steady roll structure may be realized for $\mathrm{Ra}>10^{4}$. We did not observe six or more rolls in the present vessel, however, a six-roll steady structure may emerge for $Q \sim 10^{4}$ according to the numerical analysis in Ref. [17]. Hence experiments at larger $Q$ values up to $10^{4}$ are expected. Hysteresis in behavior is observed at an increase and decrease in $Q$, around the value $30<\mathrm{Ra} / Q<100$, corresponding to regimes II and III. With a decrease in $Q$ at fixed $\mathrm{Ra}$, the behavior changes from the transition of roll number 5 and 4 (regime IV) to an oscillatory 4-roll (regime III), as shown in Fig. 9. With an increase in $Q$ at the same Ra, isotropic flow (regime I) does not change to an oscillatory roll but changes to the transition of roll numbers 3 and 4 (regime II) and to regime IV at large $Q$. In other words, regime II is a halfway state for making up an aligned roll structure in the direction of the magnetic field, while regime III is a collapsing state of roll-like structure formed under a large $Q$ value. In the emergence of regime IV, little hysteresis is observed in the present experiments. However, for the precise study of hysteresis, experiments below the detection limit of UVP measurement and experiments with larger $Q$ values are expected. Geometry and aspect ratio of a vessel are also important factors for the convective flow behavior (see reviews in Refs. [28,31]). We do not observe any repetitions of roll number transitions (including flow reversals) in a narrower vessel with a 4:1:1 length ratio ( 1 is the height), as reported in Refs. [21,22]. It may be necessary for the geometry of a vessel to have enough horizontal scales in both directions to induce the skewed-varicose instability and resulting roll number transitions. It is an interesting topic to study the roll number transitions in vessels having larger aspect ratio.

\section{CONCLUSION}

Convection patterns of a liquid metal under the influence of an imposed horizontal magnetic field were studied. The results were summarized as a regime diagram (Fig. 5), which presented five flow regimes depending on $\mathrm{Ra}$ and $Q$. The two extreme regimes are (I) developed turbulence with isotropic large-scale fluctuating patterns and $(\mathrm{V})$ a steady $2 \mathrm{D}$ roll pattern, with three regimes recognizable between these: (II) weakly constrained rolls with fluctuations, (III) continuous oscillation of rolls, and (IV) repeated random roll number transitions with flow reversals. The mechanism to induce these regime transitions is the reduction of wave numbers of a pattern, as the restriction of an imposed magnetic field becomes weaker. The relation between the intrinsic roll size under a magnetic field and the geometry of the vessel is an important constraint on the flow behavior, suggesting that the locations of regime boundaries on the $Q$-Ra plane may depend on the aspect ratio of the vessel. The basic features for each regime can be understood by considerations of the Busse balloon and its instabilities.

\section{ACKNOWLEDGMENTS}

This work was supported by JSPS KAKENHI Grant No. 24244073. The authors express thanks for this support.
[1] P. A. Davidson, An Introduction to Magnetohydrodynamics (Cambridge University Press, Cambridge, 2001).

[2] S. Chandrasekhar, Hydrodynamic and Hydromagnetic Stability (Oxford University Press, Oxford, 1961).

[3] B. Lehnert and N. C. Little, Tellus 9, 97 (1957).

[4] Y. Nakagawa, Proc. R. Soc. London Ser. A 240, 108 (1957).

[5] F. H. Busse, J. Fluid Mech. 52, 97 (1972).

[6] R. M. Clever and F. H. Busse, J. Fluid Mech. 65, 625 (1974).

[7] R. M. Clever and F. H. Busse, J. Appl. Math. Phys. 29, 711 (1978).

[8] F. H. Busse and R. M. Clever, J. Fluid Mech. 91, 319 (1979).

[9] F. H. Busse and R. M. Clever, Phys. Fluids 25, 931 (1982).

[10] F. H. Busse and R. M. Clever, J. Theor. Appl. Mech. 2, 495 (1983).

[11] S. Fauve, C. Laroche, and A. Libchaber, J. Phys. Lett. 42, L455 (1981).

[12] A. Libchaber, S. Fauve, and C. Laroche, Physica D 7, 73 (1983).
[13] S. Fauve, C. Laroche, and A. Libchaber, J. Phys. Lett. 45, L101 (1984).

[14] A. Chiffaudel, B. Perrin, and S. Fauve, Phys. Rev. A 39, 2761 (1989).

[15] J. M. Aurnou and P. L. Olson, J. Fluid Mech. 430, 283 (2001).

[16] U. Burr and U. Müller, Phys. Fluids 13, 3247 (2001).

[17] U. Burr and U. Müller, J. Fluid Mech. 453, 345 (2002).

[18] Y. Nakagawa, Proc. R. Soc. London Ser. A 249, 138 (1959).

[19] In Ultrasonic Doppler Velocity Profiler for Fluid Flow, edited by Y. Takeda, Fluid Mechanics and its Applications Vol. 101 (Springer Japan, Tokyo, 2012).

[20] T. Yanagisawa, Y. Yamagishi, Y. Hamano, Y. Tasaka, M. Yoshida, K. Yano, and Y. Takeda, Phys. Rev. E 82, 016320 (2010).

[21] T. Yanagisawa, Y. Yamagishi, Y. Hamano, Y. Tasaka, K. Yano, J. Takahashi, and Y. Takeda, Phys. Rev. E 82, 056306 (2010). 
[22] T. Yanagisawa, Y. Yamagishi, Y. Hamano, Y. Tasaka, and Y. Takeda, Phys. Rev. E 83, 036307 (2011).

[23] K. Okada and H. Ozoe, J. Heat Transfer 114, 107 (1992).

[24] Y. Tasaka, Y. Takeda, and T. Yanagisawa, Flow Meas. Instrum. 19, 131 (2008).

[25] F. H. Busse and R. M. Clever, J. Fluid Mech. 102, 75 (1981).

[26] R. Krishnamurti, J. Fluid Mech. 42, 295 (1970).

[27] R. Krishnamurti and L. N. Howard, Proc. Natl. Acad. Sci. USA 78, 1981 (1981).

[28] G. Ahlers, S. Grossmann, and D. Lohse, Rev. Mod. Phys. 81, 503 (2009).

[29] G. K. Batchelor, I. D. Howells, and A. A. Townsend, J. Fluid Mech. 5, 134 (1959).

[30] M. C. Cross and P. C. Hohenberg, Rev. Mod. Phys. 65, 851 (1993).
[31] E. Bodenschatz, W. Pesch, and G. Ahlers, Annu. Rev. Fluid Mech. 32, 709 (2000).

[32] G. E. Willis, J. W. Deardorff, and R. C. J. Somerville, J. Fluid Mech. 54, 351 (1972).

[33] A. V. Getling, Rayleigh-Bénard Convection: Structures and Dynamics (World Scientific, Singapore, 1998).

[34] F. H. Busse and R. M. Clever, Phys. Rev. A 40, 1954 (1989).

[35] F. H. Busse, O. Brausch, M. Jaletzky, and W. Pesch, in Intermittency in Turbulent Flows, edited by J. C. Vassilicos (Cambridge University Press, Cambridge, 2001).

[36] F. H. Busse and E. W. Bolton, J. Fluid Mech. 146, 115 (1984).

[37] F. H. Busse, M. Kropp, and M. Zaks, Physica D 61, 94 (1992).

[38] H.-W. Xi, X.-J. Li, and J. D. Gunton, Phys. Rev. E 62, 7909 (2000). 